\title{
Reducción de la mortalidad mediante una mejor detección de la hipertensión y los problemas con el alcohol en atención primaria de salud en España
}

\section{Reduction of mortality following better detection of hypertension and alcohol problems in primary health care in Spain}

\author{
JÜRGEN REHM*,**,***,****,*****; GERRIT GMEL*******,*******; CRISTINA SIERRA********,*********; \\ ANTONI GUAL $* * * * * * * * *, * * * * * * * * * *, * * * * * * * * * * *$. \\ * Centro de Adicciones y Salud Mental, Toronto, Canadá; ** Dalla Lana School of Public Health, Universidad de Toronto, \\ Toronto, Canadá; *** Instituto de Ciencias Médicas, Universidad de Toronto, Toronto, Canadá; **** Departamento de Psiquiatría, \\ Universidad de Toronto, Toronto, Canadá; ***** Instituto de Psicología Clínica y Psicoterapia y Centro de Epidemiología Clínica \\ y Estudios Longitudinales (CELOS), Technische Universität Dresden, Alemania; ****** Grupo de Investigación sobre Sistemas de \\ Implantes, Centro Nacional de Tecnologías de la Información y la Comunicación, Eveleigh, Australia; ******* Escuela de Ingeniería \\ Eléctrica y de Telecomunicaciones, Universidad de Nueva Gales del Sur, Australia; ******** Unidad de Hipertensión y Riesgo \\ Vascular. Departamento de Medicina Interna. Hospital Clinic. Universidad de Barcelona, Barcelona, España (Cataluña); ********* \\ Instituto de Investigaciones Biomédicas August Pi i Sunyer (IDIBAPS), Barcelona, España (Cataluña); ********** Unidad de \\ Adicciones, Departamento de Psiquiatría, Instituto de Neurociencias, Hospital Clinic, Barcelona, España (Cataluña); ************ \\ Red de Trastornos Adictivos (RTA - RETICS), Barcelona, España (Cataluña).
}

\section{Resumen}

Se estudian mediante una simulación los potenciales beneficios que puede comportar una mejora en la detección y tratamiento de la hipertensión y de los problemas relacionados con el alcohol. Los resultados muestran que si el 50\% de los varones españoles entre $40 \mathrm{y}$ 64 años que desconocen que padecen hipertensión fuesen detectados y recibiesen tratamiento; y si en el 50\% de los varones hipertensos se realizase el cribado de consumo alcohólico y recibieran consejo para la reducción de consumos o tratamiento cuando procediera, el porcentaje de hipertensión no controlada descendería del $61,2 \%$ al $55,9 \%$ (una reducción del 8,6\%). Un tercio del efecto es atribuible a la intervención sobre el alcohol. De forma similar, las mismas intervenciones en mujeres de los mismos grupos etarios implicarían una reducción del 7,4\% de la hipertensión no controlada $(40 \%$ debido a la intervención sobre alcohol). La reducción de la presión arterial en la población permitiría evitar 412 muertes prematuras por patología cardiovascular (346 varones y 66 mujeres) anualmente. Una mejor detección de la hipertensión y el cribado de consumos alcohólicos con las consiguientes intervenciones resultarían en una marcada reducción de la hipertensión no controlada y de las muertes de origen cardiovascular.

Palabras clave: Presión arterial; Hipertensión; Consumo de alcohol; Trastornos por consumo de alcohol; Atención primaria de salud; Cribado; Detección; Intervención; Simulación.

\begin{abstract}
Through a simulation study, we estimated the potential effects of better detection of hypertension and improved screening for alcohol problems with subsequent interventions. Results showed that if $50 \%$ of Spanish males between 40 and 64 years of age who are currently unaware of their hypertension become aware of their condition and receive the usual treatment, and $50 \%$ of these males with hypertension are screened for alcohol and are treated for hazardous drinking or alcohol use disorders, then the percentage of uncontrolled hypertension among men with hypertension decreases from $61.2 \%$ to $55.9 \%$, i.e. by $8.6 \%$, with about $1 / 3$ of the effect due to the alcohol intervention. For women, likewise, these interventions would decrease the percentage of women in the same age group with uncontrolled hypertension by $7.4 \%$ (about $40 \%$ due to the alcohol intervention). The reduction of blood pressure in the population would avoid 412 premature CVD deaths (346 in men, 66 in women) within one year. Therefore, better detection of hypertension and screening for alcohol with subsequent interventions would result in marked reductions of uncontrolled hypertension and CVD mortality.

Keywords: Blood pressure; Hypertension; Alcohol use; Alcohol use disorders; Primary health care; Screening; Detection; Intervention, simulation.
\end{abstract}




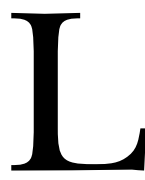
as principales enfermedades no transmisibles fueron la causa de más de la mitad de los fallecimientos a nivel mundial en el 2012 (38 de casi 56 millones; World Health Organization, 2014; Estadísticas Sanitarias Mundiales de la OMS: http://www.who.int/healthinfo/global_burden_disease/estimates/en/index 1 . html). Se ha implementado un plan de acción global con el fin de reducir esta carga, fijando un objetivo general de una reducción relativa del $25 \%$ del riesgo de muerte prematura debido a enfermedades cardiovasculares (ECV), cáncer, diabetes, o enfermedades respiratorias crónicas (World Health Organization, 2013), además de objetivos específicos para los factores de riesgo principales. Estos objetivos incluyen la hipertensión y el uso abusivo del alcohol, con reducciones previstas del $25 \%$ y el 10\%, respectivamente. Ambos factores de riesgo guardan una estrecha relación entre dosis-respuesta; i.e., cuanto mayor el consumo de alcohol, más elevada la presión arterial (PA) (Briasoulis, Agarwal, y Messerli, 2012; Taylor et al., 2009). Se ha mostrado que el efecto del alcohol es causal: aumentos y reducciones en el consumo de alcohol han resultado en cambios posteriores de presión arterial en estudios de observación y en ensayos clínicos controlados (para un repaso general: O’Keefe, Bhatti, Bajwa, DiNicolantonio, y Lavie, 2014; Xin et al., 2001; también Saunders, 1987 para una revisión inicial, incluyendo recomendaciones clínicas). La relación entre dosis-respuesta no es lineal, dado que la asociación se refuerza (es más pronunciada) a niveles más elevados de consumo de alcohol. Por lo tanto, es importante el impacto del consumo de alcohol alto y de los trastornos de consumo de alcohol en el aumento de la presión arterial y en la hipertensión (Rehm et al., 2015b; Saunders, Beevers, y Paton, 1979; Saunders, Paton, y Beevers, 1981; Taylor et al., 2009).

A la luz del impacto importante de la ingesta de alcohol abusiva en la hipertensión, no es ninguna sorpresa que el cribado, seguimiento y posteriores intervenciones en relación a este factor de riesgo ya ha sido recomendado en la década de los años 80 para controlar la hipertensión (Saunders, 1987); y en la actualidad la reducción del consumo de alcohol está incluido en los cambios recomendados del estilo de vida en el tratamiento de la hipertensión (Mancia et al., 2013a; Mancia et al., 2013b). No obstante, aparentemente el cribado de consumo alcohólico aún no es habitual de la práctica general de gestión de la hipertensión en atención primaria de salud. De hecho, el consumo de alcohol en general o el consumo abusivo en particular raramente se mencionan como temas a tratar en atención primaria, independientemente de la hipertensión (Brotons et al., 2012; Drummond et al., 2013; para España véase también http://www.papps.es/suplemento_ap_09.php ). A pesar de la práctica actual, atención primaria parece ser el entorno natural para el cribado y la intervención en problemas de hipertensión relacionados con el alcohol, ya que la mayoría de la población busca ahí atención sanitaria para todo tipo de condiciones médicas cada año (Miller, Anton, Egan, Basile, y Nguyen, 2005; Rehm et al., 2014). Es más, la hipertensión está entre los diagnósticos más frecuentes -por no decir que es el más frecuente- en atención primaria en muchos de los países desarrollados (e.g., Minas, Koukosias, Zintzaras, Kostikas, y Gourgoulianis, 2010; Ministerio de Sanidad, Servicios Sociales e Igualdad, 2010; Wändell et al., 2013), y es una enfermedad crónica que motiva la visita regular de los pacientes a sus proveedores de servicios sanitarios.

Por ello, el objetivo de nuestra aportación fue modelar lo que pasaría si:

- hubiese un aumento de la tasa de conocimiento de la hipertensión (véase Banegas et al., 2012; Catalá-López, Ridao, Sanfélix-Gimeno, y Peiró, 2013; Llisterri et al., 2012 para el nivel actual de conocimiento y control de la hipertensión en España).

- se introdujese el cribado de alcohol mediante intervenciones breves enfocadas a la ingesta de alcohol de riesgo y perjudicial y tratamientos formales para los trastornos de uso de alcohol (Babor, Higgins-Biddle, Saunders, y Monteiro, 2001; Room, Babor, y Rehm, 2005).

Las consecuencias objeto del modelo incluyen la distribución de la presión arterial sistólica y los riesgos de tener enfermedades cardiovasculares asociadas a la hipertensión (Singh et al., 2013).

\section{Métodos}

\section{Exposición de la hipertensión en España}

Se modelaron las distribuciones de la presión arterial entre personas que padecen hipertensión en España en base a los resultados de Banegas y sus colegas (Banegas et al., 2012; véase también Catalá-López et al., 2013; Llisterri et al., 2012). En el estudio de Banegas (Banegas et al., 2012), la presión arterial se midió por parte de personal formada, aplicando procedimientos estandarizados y utilizando dispositivos automáticos. Se realizaron dos lecturas de la PA, separadas por 90 minutos. Para cada conjunto de lecturas, la PA se midió 3 veces, en intervalos de entre 1-2 minutos, después de permanecer sentado durante al menos 3 minutos. En el análisis, la PA se calculó como la media de $\geq 3$ de las últimas 5 lecturas. Restringimos el modelado a la franja de edad de entre 40-64 años, dado que en este grupo de edad ya existe una prevalencia considerable de hipertensión, con menos conocimiento del mismo que en grupos de edades mayores (Banegas et al., 2012). Además, la prevalencia de la ingesta de alcohol de riesgo y perjudicial y de trastornos de uso de alcohol es alta en este grupo de edad (Rehm et al., 2015a; Rehm et al., 2014): un estudio representativo de más de 13,000 pacientes de atención primaria en 6 países de la Unión Europea halló la prevalencia siguiente: consumo peligroso de alcohol y otros problemas relacionados con el alcohol, indicativos de la necesidad de una intervención 
breve: mujeres 14,1\%-16,1\%; hombres 14,2\%-19,8\%; la dependencia del alcohol indicativo de la necesidad de terapia: mujeres 3,9\%-5,8\%; hombres $11,1 \%-16,7 \%$ ).

\section{Modelo de distribución de la presión arterial: La "campana de Gauss"}

Con el fin de estimar el cambio en la distribución de la presión arterial en un subgrupo de la población de personas con hipertensión, se diseñó una nueva distribución como alternativa a la distribución normal simple. Esta distribución, que denominamos la "campana de Gauss", es un intento para modelar la distribución asimétrica de la presión arterial entre personas con hipertensión (Pater 2005).

Se diseñó la forma de dicha campana según las reglas siguientes:

1. La forma de la campana se compone de una mitad de una distribución normal a la derecha y a la izquierda de su modus.

2. La desviación estándar de la distribución normal a la derecha de la campana es el doble de la otra mitad.

3. Las dos mitades de la distribución normal se multiplican por otras constantes para dar una distribución continua.

En base a estas suposiciones, es posible obtener mediante ingeniería las distribuciones normales necesarias si se conocen la media y la desviación estándar general de la campana final. Por lo tanto, es posible obtener una campana análoga a las medias y las desviaciones estándares de otras encuestas $\mathrm{u}$ otros datos.

La desviación estándar de la distribución normal a la izquierda del modus de la campana $\mathrm{s}_{\text {izquierda }}$, el modus de la campana, la media de la campana, $\mu$, y la desviación estándar de la campana, $s_{\text {campana }}$, se entrelazan mediante las expresiones que siguen:

$$
\begin{aligned}
& \text { Modus }=\mu-\sqrt{\frac{2}{\pi}} \cdot \sigma_{\text {izquierda }} \\
& \sigma_{\text {campana }}{ }^{2}=\mu^{2}+\text { Modus }^{2}+2 \cdot \text { Modus } \cdot \sqrt{\frac{2}{\pi}} \cdot \sigma_{\text {izquierda }}+3 \cdot \\
& \sigma_{\text {izquierda }}{ }^{2}-2 \cdot \text { Media } \cdot\left(\text { Modus }+\sqrt{\frac{2}{\pi}} \cdot \sigma_{\text {izquierda }}\right)
\end{aligned}
$$

Validamos la campana mediante la reproducción de las distribuciones actuales de la presión arterial entre personas que padecen hipertensión (controlada y no controlada) en Finlandia (Koskinen, Lundqvist, y Ristiluoma, 2012; Laatikainen et al., 2013), Alemania (Neuhauser, Thamm, y Ellert, 2013), España (Banegas et al., 2012; Catalá-López et al., 2013; Llisterri et al., 2012) y el Reino Unido (Joffres et al., 2013).

\section{Modelaje de los efectos del tratamiento y la intervención}

Las expresiones dadas arriba nos permiten crear una campana para cualquier media y desviación estándar. Para estimar los efectos de las intervenciones, se crearon un millón de muestras en base a la campana y se aplica una disminución proporcional de la presión arterial a un subconjunto de las muestras, según el porcentaje de pacientes que padecen hipertensión que reciben su intervención correspondiente.

En líneas generales, se requiere de tres pasos para comparar el estado actual con el escenario ideal en el cual todos los pacientes con hipertensión pasan por un cribado y reciben intervención para su presión arterial alta (principalmente medicamentos), y donde las personas con problemas relacionados con el alcohol reciben intervenciones adicionales, sean intervenciones breves (Kaner et al., 2007) o tratamientos formales, incluyendo farmacoterapia.

1. Se crea una campana inicial con la media y la desviación estándar conocida actualmente de la presión arterial alta entre personas que padecen hipertensión.

2. Se evalúan los efectos del cribado sistemático al atribuir a todas las personas con hipertensión la presión arterial media del grupo de pacientes que conocían y eran tratados por su presión arterial alta (en base a los datos empíricos, incluyendo a los que no llegaron a controlar su hipertensión a raíz de la intervención).

3. Por último, se evalúa el efecto de las intervenciones breves y el tratamiento formal por trastornos por consumo de alcohol mediante la disminución de la presión arterial de un subconjunto seleccionado aleatoriamente de la distribución de la campana del paso 2. Dicho subconjunto se seleccionó para reflejar la prevalencia de personas con ingesta de alcohol de riesgo y trastornos de uso de alcohol entre personas con hipertensión (Rehm et al., 2014) especificados arriba. El tamaño de esta disminución fue modelado en base al meta-análisis de Xin (Xin et al., 2001): usando el efecto medio de todas las intervenciones usadas por Xin y sus colegas (2001) para el consumo de alcohol de riesgo, y usando el efecto especificado para las terapias formales para el efecto de las terapias sobre la dependencia del alcohol.

El análisis principal presupone una reducción del $50 \%$ de las personas que desconocen que padecen hipertensión y tasas de cobertura de las intervenciones para problemas con el alcohol también del 50\%. También, realizamos dos análisis de sensibilidad: en el primero, supusimos tasas de cobertura de las intervenciones para problemas con el alcohol del $100 \%$, y en el segundo tasas de cobertura para hipertensión y alcohol del $100 \%$. Aunque los últimos objetivos parecen 
difíciles de lograr, nos dan el efecto máximo que se podría obtener mediante las intervenciones sugeridas.

\section{Modelaje de los efectos de la distribución cambiada de presión arterial sobre las enfermedades cardiovasculares}

Con el fin de estimar el número de fallecimientos evitados por las intervenciones aquí descritas, debemos comparar las distribuciones de la presión arterial antes y después de las intervenciones en combinación con las funciones de riesgos relativos asociadas a la presión arterial. Se supone, además, que las personas que no padecen hipertensión tienen un riesgo relativo de 1 .

En los casos en que las distribuciones de presión arterial antes y después de las intervenciones son conocidas, los fallecimientos evitados se computan de la siguiente forma:

\section{FallecimientosEvitados}

$=\frac{\int P_{T A_{\text {DespuésInt }}}(P A) * R R(P A) d T A-\int P_{T A_{\text {AntesInt }}}(P A) * R R(P A) d P A}{P_{\text {presiónnormal }}+\int P_{T A_{\text {AntesInt }}}(P A) * R R(P A) d P A}$

Donde $\mathrm{P}_{\text {presiónnormal }}$ es la proporción de personas que no padecen hipertensión, $\mathrm{P}_{\text {TADespuésInt }}(\mathrm{PA})$ es la distribución de presión arterial (PA) después de todas las intervenciones, $\mathrm{P}_{\text {TAAntesint }}(\mathrm{PA})$ es la distribución de presión arterial (PA) antes de cualquier intervención, y $\mathrm{RR}(\mathrm{PA})$ es el riesgo relativo de fallecimiento por una enfermedad dada con presión arterial PA.

En nuestro caso, la distribución de PA final se ha estimado usando 1 millón de muestras. La integral, por lo tanto, fue sustituida por el valor medio de la función de riesgo relativo aplicado a cada muestra. Los datos sobre mortalidad en España se tomaron de las Estadísticas Sanitarias Mundiales 2012 de la OMS (http://www.who.int/healthinfo/global_burden_disease/en/).

\section{Resultados}

\section{Resultados principales para la reducción de hiperten- sión no controlada}

Tabla 1 muestra los resultados de la proporción de personas con la presión arterial controlada y no controlada después de ambas intervenciones descritas arriba. Si el $50 \%$ de los hombres españoles con edades entre los 40 y los 64 años que actualmente desconoce su hipertensión puede saberlo y recibir intervenciones para cambiar la distribución de su presión arterial; y si el $50 \%$ de las personas con hipertensión y problemas relacionados con el alcohol o trastornos por consumo de alcohol recibe intervenciones (sean intervenciones breves para la ingesta de alcohol de riesgo o perjudicial, y para trastornos de uso de alcohol), el porcentaje de hipertensión no controlada entre hombres con hipertensión disminuiría del $61,2 \%$ al 55,9\%, i.e., un 8,6\%. Esto equivale a una reducción de hombres con edades entre los 40 y los 64 años con hipertensión no controlada en la población general en 2,2 puntos porcentuales (de 25,7\% a $23,5 \%)$. En nuestro caso, la hipertensión controlada se define como una presión arterial sistólica por debajo de 140 $\mathrm{mm} \mathrm{Hg}$. Un tercio del efecto es atribuible a la intervención sobre el alcohol.

De forma similar, estas intervenciones disminuirían el porcentaje de mujeres en el mismo grupo de edad con hipertensión no controlada en $7,4 \%$, reduciendo la proporción de dichas mujeres en la población general de 17,8\% a $16,5 \%$, i.e., 1,3 puntos porcentuales. Un $40 \%$ del efecto es atribuible a la intervención sobre el alcohol.

Tabla 1. Cambios en la presión arterial sistólica entre personas con hipertensión (controlada y no controlada) en España después de dos intervenciones hipotéticas (basado en Banegas et al., 2012)

\begin{tabular}{|c|c|c|c|c|c|c|c|c|}
\hline & \multicolumn{4}{|c|}{ Mujeres con edades entre los 40-64 años } & \multicolumn{4}{|c|}{ Hombres con edades entre los 40-64 años } \\
\hline & $\begin{array}{c}\text { Presión } \\
\text { arterial } \\
\text { sistólica } \\
\text { media (PA) }\end{array}$ & $\begin{array}{c}\% \\
\text { controlado }^{\&} \\
\text { entre } \\
\text { hipertensos }\end{array}$ & $\begin{array}{l}\text { Aumento } \\
\text { de control } \\
\text { delta\% }\end{array}$ & $\begin{array}{l}\text { PA sistólica } \\
\geq 140 \mathrm{~mm} \\
\text { Hg en la } \\
\text { población }\end{array}$ & $\begin{array}{c}\text { PA } \\
\text { sistólica } \\
\text { media }\end{array}$ & $\begin{array}{c}\% \\
\text { controlado } \\
\text { entre } \\
\text { hipertensos }\end{array}$ & $\begin{array}{l}\text { Aumento } \\
\text { de control } \\
\text { delta\% }\end{array}$ & $\begin{array}{l}\text { PA sistólica } \\
\geq 140 \mathrm{~mm} \\
\text { Hg en la } \\
\text { población }\end{array}$ \\
\hline Antes & 146,0 & 40,4 & - & 17,8 & 146,8 & 38,8 & - & 25,7 \\
\hline $\begin{array}{l}\text { Después de aumentar } \\
\text { el conocimiento }(50 \%)\end{array}$ & 144,8 & 43,0 & 2,6 & 17,0 & 145,3 & 42,2 & 3,5 & 24,2 \\
\hline $\begin{array}{l}\text { Después de aumentar } \\
\text { el conocimiento ( } 50 \%) \\
\text { e intervenciones sobre } \\
\text { el alcohol (50\%) }\end{array}$ & 144,1 & 44,8 & 4,4 & 16,5 & 144,5 & 44,1 & 5,3 & 23,5 \\
\hline $\begin{array}{l}\text { Proporción del efecto } \\
\text { atribuible a la intervención } \\
\text { sobre el alcohol }\end{array}$ & $40,5 \%$ & & & $38,5 \%$ & 33,5 & & & $31,8 \%$ \\
\hline
\end{tabular}

Nota. \& definido como presión arterial sistólica z 140 mm HG. 
Tabla 2. Beneficios esperados respecto de la mortalidad en un año dadas las intervenciones vía la reducción de presión arterial (basado en Singh et al., 2013)

\begin{tabular}{lcccc}
\hline & \multicolumn{2}{c}{ Mujeres con edades entre los $\mathbf{4 0 - 6 4}$ años } & \multicolumn{2}{c}{ Hombres con edades entre los 40-64 años } \\
\cline { 2 - 5 } & $\begin{array}{c}\text { Número de } \\
\text { fallecimientos evitados }\end{array}$ & $\begin{array}{c}\text { \% de todos los } \\
\text { fallecimientos }\end{array}$ & $\begin{array}{c}\text { Número de } \\
\text { fallecimientos evitados }\end{array}$ & $\begin{array}{c}\text { \% de todos los } \\
\text { fallecimientos }\end{array}$ \\
\hline Cardiopatía isquémica & 20 & $2,5 \%$ & 180 & $4,2 \%$ \\
\hline Cardiopatía hipertensiva & 9 & $9,9 \%$ & 33 & $14,3 \%$ \\
\hline Cardiopatía reumática & 1 & $0,7 \%$ & 1 & $1,2 \%$ \\
\hline Endocarditis & 1 & $0,7 \%$ & 24 & $6,2 \%$ \\
\hline Ictus & 6 & $4,3 \%$ & 66 & $7,7 \%$ \\
\hline Hemorragia intracraneal & 25 & $5,1 \%$ & 36 & $2,6 \%$ \\
\hline Otras ECV & 8 & $1,5 \%$ & 346 & $4,5 \%$ \\
\hline Total & 66 & $3,0 \%$ & & 76 \\
\hline
\end{tabular}

Nota. Los efectos fueron modelados para el 2012. Incluyen reducciones de la mortalidad en un año en base al efecto de ambas intervenciones en la presión arterial.

Tabla 3. Cambios en la presión arterial sistólica entre personas con hipertensión (controlada y no controlada) en España después de dos intervenciones hipotéticas (Banegas et al., 2012) - análisis de sensibilidad

Suposición: $50 \%$ de los desconocedores -> conocedores;

$100 \%$ de las personas con problemas relacionados con el alcohol reciben intervención.

\begin{tabular}{|c|c|c|c|c|c|c|c|c|}
\hline & \multicolumn{4}{|c|}{ Mujeres con edades entre los 40-64 años } & \multicolumn{4}{|c|}{ Hombres con edades entre los 40-64 años } \\
\hline & $\begin{array}{c}\text { Presión } \\
\text { arterial } \\
\text { sistólica } \\
\text { media (PA) }\end{array}$ & $\begin{array}{c}\% \\
\begin{array}{c}\% \\
\text { controlado\& } \\
\text { entre }\end{array} \\
\text { hipertensos }\end{array}$ & $\begin{array}{l}\text { Aumento } \\
\text { de control } \\
\text { delta\% }\end{array}$ & $\begin{array}{l}\text { PA sistólica } \\
\geq 140 \mathrm{~mm} \\
\text { Hg en la } \\
\text { población }\end{array}$ & $\begin{array}{c}\text { PA } \\
\text { sistólica } \\
\text { media }\end{array}$ & $\begin{array}{c}\% \\
\text { controlado }^{\&} \\
\text { entre } \\
\text { hipertensos }\end{array}$ & $\begin{array}{l}\text { Aumento } \\
\text { de control } \\
\text { delta\% }\end{array}$ & $\begin{array}{l}\text { PA sistólica } \\
\geq 140 \mathrm{~mm} \\
\text { Hg en la } \\
\text { población }\end{array}$ \\
\hline Antes & 146,0 & 40,4 & - & 17,8 & 146,8 & 38,8 & - & 25,7 \\
\hline $\begin{array}{l}\text { Después de aumentar el } \\
\text { conocimiento }(50 \%)\end{array}$ & 144,8 & 43,0 & 2,6 & 17,0 & 145,3 & 42,2 & 3.5 & 24,2 \\
\hline $\begin{array}{l}\text { Después de aumentar el } \\
\text { conocimiento (50\%) e } \\
\text { intervenciones sobre el } \\
\text { alcohol (100\%) }\end{array}$ & 143,3 & 46,4 & 6,0 & 16,0 & 143,6 & 45,9 & 7.1 & 22,7 \\
\hline $\begin{array}{l}\text { Proporción del efecto } \\
\text { atribuible a la intervención } \\
\text { sobre el alcohol }\end{array}$ & $55,6 \%$ & 56 & & $55,6 \%$ & $53,1 \%$ & & & $50,0 \%$ \\
\hline
\end{tabular}

Nota. \& definido como presión arterial sistólica $\geq 140$ mm HG.

Suposición: $100 \%$ de los desconocedores -> conocedores;

$100 \%$ de las personas con problemas relacionados con el alcohol reciben intervención.

\begin{tabular}{|c|c|c|c|c|c|c|c|c|}
\hline & \multicolumn{4}{|c|}{ Mujeres con edades entre los 40-64 años } & \multicolumn{4}{|c|}{ Hombres con edades entre los 40-64 años } \\
\hline & $\begin{array}{c}\text { Presión } \\
\text { arterial } \\
\text { sistólica } \\
\text { media (PA) }\end{array}$ & $\begin{array}{c}\% \\
\text { controlado\& } \\
\text { entre } \\
\text { hipertensos }\end{array}$ & $\begin{array}{l}\text { Aumento } \\
\text { de control } \\
\text { delta\% }\end{array}$ & $\begin{array}{l}\text { PA sistólica } \\
\geq 140 \mathrm{~mm} \\
\text { Hg en la } \\
\text { población }\end{array}$ & $\begin{array}{c}\text { PA } \\
\text { sistólica } \\
\text { media }\end{array}$ & $\begin{array}{c}\% \\
\text { controlado\& } \\
\text { entre } \\
\text { hipertensos }\end{array}$ & $\begin{array}{l}\text { Aumento } \\
\text { de control } \\
\text { delta\% }\end{array}$ & $\begin{array}{l}\text { PA sistólica } \\
\geq 140 \mathrm{~mm} \\
\text { Hg en la } \\
\text { población }\end{array}$ \\
\hline Antes & 146,0 & 40,4 & - & 17,8 & 146,8 & 38,8 & - & 25,7 \\
\hline $\begin{array}{l}\text { Después de aumentar el } \\
\text { conocimiento (100\%) }\end{array}$ & 143,7 & 45,5 & 5,1 & 16,2 & 143,7 & 45,8 & 7.0 & 22,8 \\
\hline $\begin{array}{l}\text { Después de aumentar el } \\
\text { conocimiento (100\%) e } \\
\text { intervenciones sobre el } \\
\text { alcohol (100\%) }\end{array}$ & 142,2 & 49,0 & 8,6 & 15,2 & 142,1 & 49,3 & 10.5 & 21,3 \\
\hline $\begin{array}{l}\text { Proporción del efecto } \\
\text { atribuible a la intervención } \\
\text { sobre el alcohol }\end{array}$ & $39,5 \%$ & & & $38,5 \%$ & $34,0 \%$ & & & $34,1 \%$ \\
\hline
\end{tabular}

Nota. \& definido como presión arterial sistólica $\geq 140$ mm HG. 


\section{Reducción de la mortalidad de ECV}

En general, en al plazo de un año, podrían evitarse 412 de los 9,912 fallecimientos atribuibles a enfermedades cardiovasculares en el grupo de edad de 40-64 años (datos basados en el 2012). Una mayoría abrumadora de estos fallecimientos sería de hombres, y en cardiopatía isquémica, seguido por ictus.

\section{Análisis de sensibilidad}

Además del análisis principal, realizamos 2 análisis de sensibilidad para averiguar cómo cada cambio afectó el resultado global. El primer análisis supuso que el $50 \%$ de los españoles con edades entre los 40 y los 64 años que desconoce su hipertensión lo llega a saber y, por consiguiente, cambió la distribución de su presión arterial, y que todas las personas con problemas relacionados con el alcohol o trastornos por uso de alcohol fueron objeto de intervención.

El segundo análisis supuso que todos los españoles con edades entre los 40 y los 64 años que desconoce su hipertensión fueron incluidos en la distribución de las personas que saben que padecen hipertensión y que, además, todas las personas con problemas relacionados con el alcohol o trastornos por uso de alcohol fueron objeto de intervención.

Para cada análisis de sensibilidad, los cálculos fueron nuevamente separados en 2 pasos, como en el análisis principal. Tabla 3 muestra un resumen de los resultados.

\section{Discusión}

En general, se pudo mostrar que las intervenciones sobre el alcohol en pacientes de atención primaria con hipertensión, i.e., intervenciones breves enfocadas a la ingesta de alcohol de riesgo y tratamiento o derivación a tratamiento especializado para trastornos por uso de alcohol (Babor et al., 2010; Babor et al., 2007) es prometedor en cuanto a los beneficios para el sistema sanitario público en lo que refiere a la disminución de los niveles altos de presión arterial y las enfermedades cardiovasculares que resultan de la misma (véase también Gual, Zarco, Colom, y Rehm, 2015). Con respecto a la reducción de los niveles de presión arterial se pudo mostrar que hasta un $17 \%$ de la hipertensión en los hombres y un $15 \%$ en las mujeres podría ser controlada si se reduce, al máximo, el desconocimiento y se inicia una intervención para problemas con el alcohol (véase los resultados del análisis de sensibilidad 2). En dicho escenario, las diferencias actuales entre hombres y mujeres también desaparecerían (para evaluaciones recientes sobre diferencias de sexo en España véase (Banegas et al., 2008; Gijón-Conde y Banegas, 2012; Ortiz Marrón et al., 2011).

También fue notable el impacto de las intervenciones sobre los fallecimientos por enfermedades cardiovasculares. Más de 400 fallecimientos atribuibles a enfermedades cardiovasculares se podrían evitar, dado el escenario principal, en un año (véase detalles en Tabla 2), principalmente en hombres. Además, y sin haber sido explícitamente modelado aquí, una reducción en el uso de alcohol se asociaría con una reducción notable a corto plazo de morbilidad y mortalidad para muchas consecuencias de enfermedades (Rehm y Roerecke, 2013; Rehm, Shield, Rehm, Gmel, y Frick, 2013; específicamente para España: Rehm, Rehm, Shield, Gmel, y Gual, 2013; Soler González, Balcells Oliveró, y Gual Solé, 2014; para un listado completo de enfermedades atribuibles al alcohol, véase Rehm et al., 2010). Para dar una idea de la magnitud de dichas reducciones como resultado de tratamiento formal: si se reduce el consumo de alcohol, incluyendo pero sin limitarse a conseguir la abstinencia, el riesgo de la mortalidad atribuible a todas las causas se reducirá en aproximadamente un $60 \%$ en total dentro de 9 años (resultados basados en un meta-análisis de todos los estudios de tratamiento con datos relevantes Roerecke, Gual, y Rehm, 2013). Estos efectos, no obstante, incluyeron los efectos sobre enfermedades cardiovasculares vía hipertensión.

En resumen, se puede subrayar el impacto de un mejor conocimiento e intervenciones sobre el alcohol. ¿Qué obstáculos impiden que el sistema actual lo consiga? Primero, el conocimiento de la hipertensión aún se considera un problema de las personas mayores. Claramente, cuanto mayor edad de la población, mejor nivel de conocimiento (Banegas et al., 2012): mientras que las dos terceras partes de las personas menores de 45 años de edad en España desconoce que padece hipertensión, la mayor parte de la población mayor de 65 años (de nuevo, aproximadamente unas dos terceras partes) sí era conocedora de su condición respecto de la enfermedad. Para los médicos de atención primaria, el cribado de hipertensión entre personas jóvenes, en particular entre hombres jóvenes con un alto consumo de alcohol (Org et al., 2011), vale la pena. Segundo, el cribado de uso de alcohol y la aplicación de intervenciones breves o tratamiento podría representar una diferencia marcada en relación al control de hipertensión. Los centros de atención primaria son los puntos ideales para la aplicación de dichos cribados e intervenciones tempranas: además de que los médicos de atención primaria reconocen los casos de consumo abusivo de alcohol y de los trastornos por consumo de alcohol (Rehm et al., 2015a), este entorno posibilita las intervenciones breves y el tratamiento para los trastornos por consumo de alcohol menos severos (Rehm et al., 2016; Rubio, Jiménez-Arriero, Martinez, Ponce, y Palomo, 2010; Segura Garcia, Gual Solé, Montserrat Mestre, Bueno Belmonte, y Colom Farran, 2006). Tercero, en caso de aplicar dichas intervenciones, la tasa de hipertensión resistente al tratamiento (para una definición véase Boswell, Pascual, y Oliveras, 2015; para España: Oliveras y de la Sierra, 2014) probablemente se reduciría (Calhoun et al., 2008; Denolle et al., 2014). Es más, las intervenciones sobre el alcohol pueden afectar la ingesta de medicamentos (Miller et al., 2005), no únicamente en personas con 
hipertensión (Grodensky, Golin, Ochtera, y Turner, 2012), y la reducción del consumo de alcohol reducirá el riesgo y la severidad de otras comorbilidades (Diaz et al., 2014; Rehm, Manthey, Struzzo, Gual, y Wojnar, 2015d; Rehm y Roerecke, 2013).

Ningún modelaje es exento de limitaciones. Primero, obtuvimos nuestros datos sobre prevalencia y medias a través de un estudio de gran tamaño con una evaluación minuciosa de hipertensión por parte de personal formada (Banegas et al., 2012). No obstante, las posibilidades de "bata blanca" o "hipertensión de estudio/oficina aislada" sólo podría excluirse mediante la toma de muestras de sangre en 24-horas, por ejemplo, como establecen las normas del Instituto Nacional de Excelencia Clínica en el Reino Unido (Mayor, 2011). Aunque nuestros datos pueden sobrestimar la prevalencia real de personas que padecen hipertensión, este efecto aparentemente no suma a más del 10\% (Banegas et al., 2015), basado en una muestra de mayor edad). Segundo, mientras que la campana ha probado mostrar la distribución de la presión arterial en España bastante bien, cualquier modelo supone una simplificación algo sesgada. Tercero, los resultados del meta-análisis de presión arterial y consecuencias cardiovasculares (Singh et al., 2013) se usaron para España, por lo tanto suponiendo que las relaciones entre los riesgos mantenían su veracidad. Esta suposición es standard en el modelado global de carga de enfermedades (Ezzati, Lopez, Rodgers, y Murray, 2004; Rehm et al., 2009); ha de ser contrastada con datos locales, siempre que sea posible (e.g., Roerecke et al., 2015). No encontramos datos para España detallando el aumento del riesgo para varias de las categorías de riesgo cardiovascular examinadas y, por lo tanto, aplicamos relaciones de riesgo globales (Singh et al., 2013). El error introducido no parece ser demasiado grande, dado que la mayoría de los estudios anteriores se realizaron en países desarrollados, y las relaciones se basan, principalmente, en mecanismos biológicos.

En general, el modelado y el análisis de sensibilidad claramente muestran que intervenciones para aumentar el conocimiento de la hipertensión y de cribado e intervención en problemas relacionados con el alcohol y en los trastornos por consumo de alcohol resultarán en reducciones significativas, en la sanidad pública, de personas con presión arterial sistólica por encima de $140 \mathrm{~mm} \mathrm{Hg}$. Atención primaria parece ser el mejor lugar para situar estas intervenciones, y es donde la hipertensión se controla en la mayoría de los casos de todas formas. Existen tres requisitos para la implementación: primero, los médicos de atención primaria han de recibir formación para implementar dichas intervenciones sobre el alcohol (Rehm et al., 2016); segundo, han de disponer de suficiente tiempo diariamente durante sus jornadas laborales para realizar intervenciones breves y tratamientos formales para la dependencia del alcohol, y tercero, han de implementarse unas estructuras de incentivos adecuadas (Anderson et al., 2014; O’Donnell et al., 2014).

\section{Reconocimientos}

Este manuscrito fue redactado durante la excedencia del primer autor del Hospital Clinic de Barcelona, España, entre enero y marzo del 2015. En parte fue inspirado por la "Mesa Redonda de Expertos: Cribado e intervención en el abuso del alcohol como herramienta para mejorar la gestión de hipertensión en atención primaria” celebrado el 6 de mayo de 2014 en Barcelona, bajo el patrocinio de Lundbeck, a la que asistieron tres de los cuatro autores (JR, CS, AG). No percibieron remuneración económica alguna por la preparación del manuscrito.

\section{Conflicto de intereses y fuentes de financiación}

Dr. Rehm ha percibido ayudas, ingresos personales y demás (es miembro de la Junta Directiva) de Lundbeck, con independencia del artículo presentado. CS ha percibido ingresos personales de Lundbeck, con independencia del artículo presentado. AG ha percibido ayudas e ingresos personales de Lundbeck, ayudas e ingresos personales de D\&A Pharma, e ingresos personales de AbbVie, con independencia del artículo presentado. No percibieron remuneración económica alguna por la preparación del manuscrito.

\section{Referencias}

Anderson, P., Wojnar, M., Jakubczyk, A., Gual, A., Segura, L., Sovinova, H., ... Kolsek, M. (2014). Managing alcohol problems in general practice in Europe: results from the European ODHIN survey of general practitioners. Alcohol and Alcoholism, 49, 531-539. doi: 10.1093/alcalc/ agu043.

Babor, T., Caetano, R., Casswell, S., Edwards, G., Giesbrecht, N., Graham, K., ... Rossow, I. (2010). Alcohol: No ordinary commodity. Research and public policy. 2nd edition. Oxford and London: Oxford University Press.

Babor, T. F., Higgins-Biddle, J. C., Saunders, J. B. y Monteiro, M. G. (2001). The Alcohol Use Disorders Identification Test Guidelines for Use in Primary Care (2nd Edition). Geneva, Switzerland: World Health Organization.

Babor, T. F., McRee, B. G., Kassebaum, P. A., Grimaldi, P. L., Ahmed, K. y Bray, J. (2007). Screening, Brief Intervention, and Referral to Treatment (SBIRT): toward a Public Health approach to the management of substance abuse. Substance Abuse, 28, 7-30.

Banegas, J. R., Graciani, A., de la Cruz-Troca, J. J., León-Muñoz, L. M., Guallar-Castillón, P., Coca, A., ... Rodríguez-Artalejo, F. (2012). Achievement of cardiometabolic goals in aware hypertensive patients in Spain: a nationwide population-based study. Hypertension, 60, 898-905. doi: 10.1161/HYPERTENSIONAHA.112.193078.

Banegas, J. R., Segura, J., de la Sierra, A., Gorostidi, M., Rodríguez-Artalejo, F., Sobrino, J., ... Ruilope, L. M. 
(2008). Gender differences in office and ambulatory control of hypertension. The American Journal of Medicine, 121, 1078-1084. doi: 10.1016/j.amjmed.2008.06.037.

Banegas, J.R., Navarro-Vidal, B., Ruilope, L.M., de la Cruz, J.J., López-García, E. y Rodríguez-Artalejo, F., Graciani, A. (2015). Trends in hypertension control among the older population of Spain from 2000 to 2001 to 2008 to 2010: role of frequency and intensity of drug treatment. Circulation. Cardiovascular Quality and Outcomes, 8, 67-76. doi: 10.1161/CIRCOUTCOMES.114.001191.

Boswell, L., Pascual, J. y Oliveras, A. (2015). Resistant hypertension: do all definitions describe the same patients? Journal of Human Hypertension. doi: 10.1038/ jhh.2014.128.

Briasoulis, A., Agarwal, V. y Messerli, F. H. (2012). Alcohol consumption and the risk of hypertension in men and women: a systematic review and meta-analysis. The Journal of Clinical Hypertension, 14, 792-798. doi: 10.1111/jch.12008.

Brotons, C., Bulc, M., Sammut, M. R., Sheehan, M., Manuel da Silva Martins, C., Björkelund, C., ... Godycki-Cwirko, M. (2012). Attitudes toward preventive services and lifestyle: the views of primary care patients in Europe. the EUROPREVIEW patient study. Family Practice 29, i168-i176. doi: 10.1093/fampra/cmr102.

Calhoun, D. A., Jones, D., Textor, S., Goff, D. C., Murphy, T. P., Toto, R. D., ... Carey, R. M. (2008). Resistant hypertension: diagnosis, evaluation, and treatment: a scientific statement from the American Heart Association Professional Education Committee of the Council for High Blood Pressure Research. Circulation, 117, e510-526. doi: 10.1161/circulationaha.108.189141.

Catalá-López, F., Ridao, M., Sanfélix-Gimeno, G. y Peiró, S. (2013). Trends of uncontrolled blood pressure in Spain: an updated meta-regression analysis. Journal of Hypertension, 31, 630-631. doi: 10.1097/HJH.0b013e32835c6d19

Denolle, T., Chamontin, B., Doll, G., Fauvel, J. P., Girerd, X., Herpin, D., ... Halimi, J. M. (2014). [Management of resistant hypertension. Expert consensus statement from the French Society of Hypertension, an affiliate of the French Society of Cardiology]. Presse Medicale, 43, 1325-1331. doi: 10.1016/j.lpm.2014.07.016.

Diaz, K. M., Booth, J. N., 3rd, Calhoun, D. A., Irvin, M. R., Howard, G., Safford, M. M., ... Shimbo, D. (2014). Healthy lifestyle factors and risk of cardiovascular events and mortality in treatment-resistant hypertension: the Reasons for Geographic and Racial Differences in Stroke study. Hypertension, 64, 465-471. doi: 10.1161/hypertensionaha.114.03565.

Drummond, C., Wolstenholme, A., DeLuca, P., Davey, Z., Donoghue, K., Elzerbi, C., ... Kaner, E. (2013). Alcohol interventions and treatments in Europe. En P. Anderson, F. Braddick, J. Reynolds y A. Gual (Eds.), Alcohol Policy in Europe: Evidence from AMPHORA, 2nd Edition (pp. 65-80). Barcelona: AMPHORA.
Ezzati, M., Lopez, A., Rodgers, A. y Murray, C. J. L. (2004). Comparative quantification of health risks. Global and regional burden of disease attributable to selected major risk factors. Geneva, Switzerland: World Health Organization.

Gijón-Conde, T. y Banegas, J. R. (2012). Cardiovascular disease in hypertension: gender differences in 100,000 clinical records. Revista Clinica Española, 212, 55-62. doi: 10.1016/j.rce.2011.07.013.

Grodensky, C. A., Golin, C. E., Ochtera, R. D. y Turner, B. J. (2012). Systematic review: effect of alcohol intake on adherence to outpatient medication regimens for chronic diseases. Journal of Studies on Alcohol and Drugs, 73, 899-910.

Gual, A., Zarco, J., Colom, J. F. y Rehm, J. (2015). Cribado precoz e intervención breve en el consumo perjudicial de alcohol para mejorar el tratamiento de la hipertensión arterial en atención primaria [Early screening and brief intervention in alcohol misuse to improve the treatment of hypertension in primary care]. Medicina Clínica, pii:S0025-7753, 00462-00465. doi: 10.1016/j.medcli.2015.07.014.

Joffres, M., Falaschetti, E., Gillespie, C., Robitaille, C., Loustalot, F., Poulter, N., ... Campbell, N. (2013). Hypertension prevalence, awareness, treatment and control in national surveys from England, the USA and Canada, and correlation with stroke and ischaemic heart disease mortality: a cross-sectional study. BMJ Open, 3, e003423. doi: 10.1136/bmjopen-2013-003423.

Kaner, E. F., Beyer, F., Dickinson, H. O., Pienaar, E., Campbell, F., Schlesinger, C., ... Burnand, B. (2007). Effectiveness of brief alcohol interventions in primary care populations. Cochrane Database of Systematic Reviews, 18 , CD004148.

Koskinen, S., Lundqvist, A. y Ristiluoma, N. (2012). Terveys, toimintakysky ja hyvinvointi Suomessa 2011. Tampere: Juvenes Print - Suomen Yliopistopaino Oy.

Laatikainen, T., Jula, A., Kastarinen, M., Salomaa, V., Borodulin, K., Harald, K., ... Vartianen, E. (2013). Verenpainetasot ja hoitotasapaino FINRISKI-tutkimusaluiella 1982-2012 [Blood pressure levels and therapeutic balance in FINRISK study areas in 1982-2012]. Suomen Laakarilehti, 68, 1803-1809.

Llisterri, J. L., Rodriguez-Roca, G. C., Escobar, C., Alonso-Moreno, F. J., Prieto, M. A., Barrios, V., ... Beato, P. (2012). Treatment and blood pressure control in Spain during 2002-2010. Journal of Hypertension, 30, 2425-2431. doi: 10.1097/HJH.0b013e3283592583.

Mancia, G., Fagard, R., Narkiewicz, K., Redon, J., Zanchetti, A., Bohm, M., ... Zannad, F. (2013a). 2013 ESH/ ESC Guidelines for the management of arterial hypertension: the Task Force for the management of arterial hypertension of the European Society of Hypertension (ESH) and of the European Society of Cardiology (ESC). Journal of Hypertension, 31, 1281-1357. doi: 10.1097/01. hjh.0000431740.32696.cc. 
Mancia, G., Fagard, R., Narkiewicz, K., Redon, J., Zanchetti, A., Böhm, M., ... Zannad, F. (2013b). Guía de práctica clínica de la ESH/ESC 2013 para el manejo de la hipertensión arterial. Revista Española de Cardiología, 66, e64.

Mayor, S. (2011). Hypertension diagnosis should be based on ambulatory blood pressure monitoring, NICE recommends. BMJ 343, d5421. doi: 10.1136/bmj.d5421.

Miller, P. M., Anton, R. F., Egan, B. M., Basile, J. y Nguyen, S. A. (2005). Excessive alcohol consumption and hypertension: clinical implications of current research. The Journal of Clinical Hypertension, 7, 346-353.

Minas, M., Koukosias, N., Zintzaras, E., Kostikas, K. y Gourgoulianis, K. I. (2010). Prevalence of chronic diseases and morbidity in primary health care in central Greece: an epidemiological study. BMC Health Services Research, 10, 252. doi: 10.1186/1472-6963-10-252.

Ministerio de Sanidad, Servicios Sociales e Igualdad. (2010). Sistema Nacional de Salud de España, 2010. Madrid, España: Ministerio de Sanidad y Servicios Sociales, Instituto de Información Sanitaria.

Neuhauser, H., Thamm, M. y Ellert, U. (2013). Blutdruck in Deutschland 2008-2011. Ergebnisse der Studie zur Gesundheit Erwachsener in Deutschland (DEGS1). Bundesgesundheitsbl, 56, 795-801.

O’Donnell, A., Anderson, P., Newbury-Birch, D., Schulte, B., Schmidt, C., Reimer, J. y Kaner, E. (2014). The impact of brief alcohol interventions in primary healthcare: a systematic review of reviews. Alcohol and Alcoholism, 49, 66-78. doi: 10.1093/alcalc/agt170.

O’Keefe, J. H., Bhatti, S. K., Bajwa, A., DiNicolantonio, J. J. y Lavie, C. J. (2014). Alcohol and cardiovascular health: the does makes the poison...or the remedy. Mayo Clinic Proceedings, 89, 382-393. doi: 10.1016/j.mayocp.2013.11.005.

Oliveras, A. y de la Sierra, A. (2014). Resistant hypertension: patient characteristics, risk factors, co-morbidities and outcomes. Journal of Human Hypertension, 28, 213217. doi: 10.1038/jhh.2013.77.

Org, E., Veldre, G., Viigimaa, M., Juhanson, P., Putku, M., Rosenberg, M., ... Laan, M. (2011). HYPEST study: profile of hypertensive patients in Estonia. BMC Cardiovascular Disorders, 11, 55. doi: 10.1186/1471-2261-11-55.

Ortiz Marrón, H., Vaamonde Martín, R. J., Zorrilla Torrás, B., Arrieta Blanco, F., Casado López, M. y Medrano Albero, M. J. (2011). Prevalence, degree of control and treatment of hypertension in the adult population of Madrid, Spain. Revista Española de Salud Pública, 85, 329338. doi: 10.1590/s1135-57272011000400002.

Pater, C. (2005). The Blood Pressure "Uncertainty Range" - a pragmatic approach to overcome current diagnostic uncertainties (II). Current Controlled Trials in Cardiovascular Medicine, 6, 5. doi: 10.1186/1468-6708-6-5.

Rehm, J., Allamani, A., Elekes, Z., Jakubczyk, A., Landsmane, I., Manthey, J., ... Wojnar, M. (2015a). General practitioners recognizing alcohol dependence: a large cross-sectional study in six European countries. Annals of Family Medicine, 13, 28-32. doi: 10.1370/afm.1742.

Rehm, J., Allamani, A., Elekes, Z., Jakubczyk, A., Manthey, J., Probst, P., ... Wojnar, M. (2015b). Alcohol dependence and treatment utilization in Europe - a representative cross-sectional study in primary care. BMC Family Practice, 16, 90. doi: 10.1186/s12875-015-0308-8.

Rehm, J., Anderson, P., Manthey, J., Shield, K.D., Struzzo, P., Wojnar, M., \& Gual, A. (2016). Alcohol use disorders in primary health care - what do we know and where do we go? Alcohol and Alcoholism, 51(4), 422-427. doi: 10.1093/alcalc/agv127.

Rehm, J., Baliunas, D., Borges, G. L., Graham, K., Irving, H. M., Kehoe, T., ... Taylor, B. (2010). The relation between different dimensions of alcohol consumption and burden of disease - An overview. Addiction, 105, 817-843. doi: 10.1111/j.1360-0443.2010.02899.x.

Rehm, J., Gmel, G., Kiefer, F., Kreutz, R., Kugler, J., Müller-Walther, M., ... Weisser, B. (2014). Verbessertes Hypertonie-Management durch Alkohol-Screening und Folgeinterventionen in der Hausarztpraxis [Improvement of management of hypertension by implementation of alcohol screening and subsequent interventions in primary practice]. Deutsche Medizinische Wochenschrift, 139, 2457-2462. doi: 10.1055/s-0034-1387417.

Rehm, J., Manthey, J., Struzzo, P., Gual, A. y Wojnar, M. (2015d). Who receives treatment for alcohol use disorders in the European Union? A cross-sectional representative study in primary and specialized health care. European Psychiatry, 30, 885-893. doi: 0.1016/j.eurpsy.2015.07.012.

Rehm, J., Mathers, C., Popova, S., Thavorncharoensap, M., Teerawattananon, Y. y Patra, J. (2009). Global burden of disease and injury and economic cost attributable to alcohol use and alcohol use disorders. Lancet, 373, 22232233. doi: 10.1016/S0140-6736(09)60746-7.

Rehm, J., Rehm, M. X., Shield, K. D., Gmel, G. y Gual, A. (2013). Alcohol consumption, alcohol dependence and related harms in Spain, and the effect of treatment-based interventions on alcohol dependence. Adicciones, 25, 11-18.

Rehm, J. y Roerecke, M. (2013). Reduction of drinking in problem drinkers and all-cause mortality. Alcohol and Alcoholism, 48, 509-513. doi: 10.1093/alcalc/agt021.

Rehm, J., Shield, K. D., Rehm, M. X., Gmel, G. y Frick, U. (2013). Modelling the impact of alcohol dependence on mortality burden and the effect of available treatment interventions in the European Union. European Neuropsychopharmacology, 23, 89-97. doi: 10.1016/j.euroneuro.2012.08.001.

Roerecke, M., Gual, A. y Rehm, J. (2013). Reduction of alcohol consumption and subsequent mortality in alcohol use disorders: systematic review and meta-analysis. Jour- 
nal of Clinical Psychiatry, 74, e1181-e1189. doi: 10.4088/ JCP.13r08379.

Roerecke, M., Shield, K. D., Higuchi, S., Yoshimura, A., Larsen, E., Rehm, M. X. y Rehm, J. (2015). Correcting estimates of alcohol-related oesophageal cancer burden in Japan: a systematic review and meta-analyses. Bulletin of the World Health Organization, 93, 329-338C. doi: 10.2471/BLT.14.142141.

Room, R., Babor, T. y Rehm, J. (2005). Alcohol and public health: a review. Lancet, 365, 519-530.

Rubio, G., Jiménez-Arriero, M. A., Martinez, I., Ponce, G. y Palomo, T. (2010). Efficacy of physician-delivered brief counseling intervention for binge drinkers. The American Journal of Medicine, 123, 72-78. doi: 10.1016/j.amjmed.2009.08.012.

Saunders, J. B. (1987). Alcohol: an important cause of hypertension. British Medical Journal, 294, 1045-1046.

Saunders, J. B., Beevers, D. G. y Paton, A. (1979). Factors influencing blood pressure in chronic alcoholics. Clinical Science, 57, 295s-298s.

Saunders, J. B., Paton, A. y Beevers, D. G. (1981). Alcohol-induced hypertension. Lancet, 2, 653-656.

Segura Garcia, L., Gual Solé, A., Montserrat Mestre, O., Bueno Belmonte, A. y Colom Farran, J. (2006). [Detection and handling of alcohol problems in primary care in Catalonia]. Atencion Primaria, 37, 484-488.

Singh, G. M., Goodarz, D., Faradfar, F., Stevens, G. A., Woodward, M., Wormser, D., ... Prospective Studies Collaboration (PSC). (2013). The age-specific quantitative effect of metabolic risk factors on cardiovascular diseases and diabetes: a pooled analyses. PLoS ONE, 8, e65174. doi: 10.1371/journal.pone.0065174.

Soler González, C., Balcells Oliveró, M. y Gual Solé, A. (2014). Alcohol related brain damage. State of the art and a call for action. Adicciones, 26, 199-207.

Taylor, B., Irving, H. M., Baliunas, D., Roerecke, M., Patra, J., Mohapatra, S. y Rehm, J. (2009). Alcohol and hypertension: gender differences in dose-response relationships determined through systematic review and meta-analysis. Addiction, 104, 1981-1990. doi: 10.1111/j.1360-0443.2009.02694.x.

Wändell, P., Carlsson, A. C., Wettermark, B., Lord, G., Cars, T. y Ljunggren, G. (2013). Most common diseases diagnosed in primary care in Stockholm, Sweden, in 2011. Family Practice, 30, 506-513. doi: 10.1093/fampra/ cmt033.

World Health Organization. (2013). Global Action Plan for the Prevention and Control of NCDs 2013-2020. Geneva, Switzerland: World Health Organization.

World Health Organization. (2014). Global status report on noncommunicable diseases 2014. Attaining the nine global noncommunicable diseases targets; a shared responsibility. Geneva, Switzerland: World Health Organization.
Xin, X., He, J., Frontini, M. G., Ogden, L. G., Motsamai, O. J. y Whelton, P. K. (2001). Effects of alcohol reduction on blood pressure: a meta-analysis of randomized controlled trials. Hypertension, 38, 1112-1117. 